\title{
Streptococcus suis Serotype 2 Biofilms Inhibit the Formation of Neutrophil Extracellular Traps
}

\author{
Fang Ma ${ }^{1}$, Li Yij ${ }^{1,2}$, Ningwei $Y u^{1}$, Guangyu $\mathrm{Wang}^{3}$, Zhe Ma ${ }^{1}$, Huixing $\operatorname{Lin}^{1}$ and \\ Hongjie Fan ${ }^{1,4 *}$ \\ ${ }^{1}$ College of Veterinary Medicine, Nanjing Agricultural University, Nanjing, China, ${ }^{2}$ College of Life Science, Luoyang Normal \\ University, Luoyang, China, ${ }^{3}$ National Center of Meat Quality and Safety Control, Nanjing Agriculture University, Nanjing, \\ China, ${ }^{4}$ Jiangsu Co-Innovation Center for Prevention and Control of Important Animal Infectious Diseases and Zoonoses, \\ Yangzhou, China
}

Invasive infections caused by Streptococcus suis serotype 2 (SS2) has emerged as a clinical problem in recent years. Neutrophil extracellular traps (NETs) are an important mechanism for the trapping and killing of pathogens that are resistant to phagocytosis. Biofilm formation can protect bacteria from being killed by phagocytes. Until now, there have only been a few studies that focused on the interactions between bacterial biofilms and NETs. SS2 in both a biofilm state and a planktonic cell state were incubated with phagocytes and NETs, and bacterial survival was assessed. DNase I and cytochalasin B were used to degrade NET DNA or suppress phagocytosis, respectively. Extracellular DNA was stained with impermeable fluorescent dye to quantify NET formation. Biofilm formation increased up to 6-fold in the presence of neutrophils,

OPEN ACCESS

Edited by:

Silvia Mercedes Uriarte,

University of Louisville, USA

Reviewed by:

Teneema Kuriakose

St. Jude Children's Research Hospital,

USA

Jennifer Angeline Gaddy, Vanderbilt University, USA

*Correspondence:

Hongjie Fan

fhj@njau.edu.cn

Received: 16 January 2017 Accepted: 06 March 2017 Published: 20 March 2017

Citation:

Ma F, Yi L, Yu N, Wang G, Ma Z, Lin H and Fan $H$ (2017) Streptococcus suis

Serotype 2 Biofilms Inhibit the

Formation of Neutrophil Extracellular

Traps.

Front. Cell. Infect. Microbiol. 7:86 doi: 10.3389/fcimb.2017.00086 and biofilms were identified in murine tissue. Both planktonic and biofilm cells induced neutrophils chemotaxis to the infection site, with neutrophils increasing by 85.1 and $73.8 \%$, respectively. The bacteria in biofilms were not phagocytized. The bactericidal efficacy of NETs on the biofilms and planktonic cells were equal; however, the biofilm extracellular matrix can inhibit NET release. Although biofilms inhibit NETs release, NETs appear to be an important mechanism to eliminate SS2 biofilms. This knowledge advances the understanding of biofilms and may aid in the development of treatments for persistent infections with a biofilm component.

Keywords: zoonosis, streptococcus toxic shock-like syndrome, NETs, bacterial biofilm, clinical therapy

\section{INTRODUCTION}

Streptococcus suis (SS) is a major swine pathogen that causes a variety of diseases such as septicemia, meningitis, and endocarditis, which lead to economic losses. S. suis serotype 2 (SS2) is considered the most pathogenic and prevalent capsular type (Wertheim et al., 2009; Kerdsin et al., 2016). People working with pigs or people who consume pork-derived products from infected animals are at risk. During the last decade, several human epidemic outbreaks were reported in Asia and all over the world (Gottschalk et al., 2010a,b; Goyette-Desjardins et al., 2014). In addition, streptococcus toxic shock-like syndrome (STSLS), a peracute infection characterized by shock and a high mortality rate, is reported to be caused by SS2, resulting in increased public health concerns worldwide (Tang et al., 2006; Gomez et al., 2014). 
Bacterial biofilms are bacterial communities and are an important mechanism for bacterial resistance to immune system pressures and antimicrobials (Bojarska et al., 2016). Most of SS2 clinical isolates can form biofilms, which contribute to persistent infection, transmission and difficulties to eradicate infection (Bojarska et al., 2016). However, little information is available on the interaction between the host immune system and SS2 biofilms (Thurlow et al., 2011; Yang et al., 2016). Neutrophil extracellular traps (NETs), which are composed of granule and nuclear constituents, are made by activated neutrophils (Brinkmann et al., 2004; Uhlmann et al., 2016). The nuclear constituents are DNA and histones; DNA is the backbone of NETs and traps the pathogens by charge interactions (Wartha et al., 2007). In the recent years, NETs have been identified as a significant antibacterial mechanism employed by neutrophils (Csomos et al., 2016). Neutrophils are observed to generate NETs upon activation with interleukin-8 (IL-8), phorbol myristate acetate (PMA), lipopolysaccharide (LPS), and various microbes (Leshner et al., 2012). NETs can disarm and kill a variety of pathogens, including GAS, S. aureus, Shigella flexneri, and fungi, by capturing the microbes and providing a high local concentration of antimicrobial granules (Brinkmann et al., 2004; Buchanan et al., 2006; May et al., 2015). NETs have been found to be abundant at in vivo sites of infection and inflammation, including in cases of the autoimmune disease systemic lupus erythematosus and a murine model of pneumococcal pneumonia (Beiter et al., 2006; Hakkim et al., 2010).

In previous studies examining the immune system response to various microorganisms, certain microbes have been shown to evade phagocytosis but become entrapped by NETs (Branzk et al., 2014). Candida albicans biofilms evade phagocytosis and impair NET formation (Johnson et al., 2016). However, the nature of bacteria and fungi is dramatically different, particularly in size and biofilm structure. Therefore, whether bacterial biofilms can stimulate NET formation is unknown and the influence of SS2 biofilms on bacterial survival in NETs is unclear and requires exploration.

Bacterial biofilm formation allows bacteria to persist in the host, making the treatment of streptococcosis challenging (Walker et al., 2005). Cases of human infections worldwide stress the lack of knowledge on the virulence and interactions with host immune cells. Our study provides further knowledge on SS2 biofilm and immune response interactions, which can lead to novel approaches to streptococcosis clinical therapy. Further understanding of host-SS2 interactions may help to explain the complex evolution of the emerging human threat.

\section{MATERIALS AND METHODS}

\section{Ethics Statement}

This study was carried out in an accordance to animal welfare standards and were approved by the Ethical Committee for Animal Experiments of Nanjing Agricultural University, China. All animal experiments accorded with the guidelines of the Animal Welfare Council of China.

\section{Bacterial Strains and Cells}

The wild-type SS2 strain ZY05719 is an isolate from Jiangsu Province and was grown in Todd-Hewitt broth (THB) medium (Difco, BD, Franklin, NJ, USA) at $37^{\circ} \mathrm{C}$ on a gently rocking shaker. The bacteria were cultured to the mid-exponential phase and were collected in media for the experiment using planktonic cells. SS2 biofilms were identified with Congo Red Agar composed of 3\% THB, 0.08\% Congo Red (Sigma Aldrich, St. Louis, Mo, USA), 0.5\% glucose (Biosharp, Anhui, China) and $1.5 \%$ agar powder. Neutrophils from the bones of mice were cultured in RPMI 1640 (Gibco-BRL, Thermo Fisher Scientific, Waltham, MA, USA) supplemented with $2 \%$ heatinactivated fetal bovine serum (FBS) at $37^{\circ} \mathrm{C}$ in $5 \% \mathrm{CO}_{2}$. RAW264.7 cells (ATCC ${ }^{\mathrm{R}}$ TIB-71 ${ }^{\mathrm{TM}}$ ) were purchased from the American Type Culture Collection (ATCC) and were cultured in Dulbecco's modified Eagle's medium (DMEM; Wisent, Canada) supplemented with $10 \% \mathrm{FBS}$ at $37^{\circ} \mathrm{C}$ in $5 \% \mathrm{CO}_{2}$.

\section{Isolation of Neutrophils from Mouse Bone Marrow}

Neutrophils were isolated from 4-week-old ICR mice as previously described with modifications (Zhao et al., 2015). Briefly, the mice were euthanized and sprayed with $70 \%$ ethanol. The bone marrow from the tibias and femurs was flushed with sterile PBS with a 20-gauge needle into a $15 \mathrm{ml}$ Falcon tube (BD Falcon), and the cells were washed by centrifugation at $400 \times \mathrm{g}$ for $10 \mathrm{~min}$. The cell pellet was resuspended in $3 \mathrm{ml}$ of PBS. A Percoll (Sigma Aldrich) density gradient was prepared in a 15 $\mathrm{ml}$ Falcon tube by the careful addition of $3 \mathrm{ml}$ of $80 \%$ Percoll followed by $3 \mathrm{ml}$ of $65 \%$ Percoll and $3 \mathrm{ml}$ of $55 \%$ Percoll. The cell suspension was overlaid carefully and centrifuged for 30 $\min$ at $1000 \times \mathrm{g}$ at room temperature. The top layer and the $55 \%$ Percoll layer were carefully aspirated and discarded. The cells at the $80 / 65 \%$ gradient interface were collected and then washed and suspended in RPMI1640 medium. A greater than $90 \%$ neutrophil purity was confirmed by Trypan blue staining and flow cytometry.

\section{Neutrophil Detection by Flow Cytometry}

Flow cytometry was performed as previously described with modification (Barletta et al., 2012). The neutrophils were stained with $0.1 \mu \mathrm{g}$ of FITC-mouse Ly6G antibody (eBioscience, San Diego, CA, USA) and $0.1 \mu \mathrm{g}$ of PE-mouse CD11b antibody (eBioscience). All of the experiments were recorded using Accuri Cflow software (BD Bioscience, CA, USA) and were analyzed using FlowJo software (Three Star, Ashland, OR, USA).

\section{Biofilm Formation In vitro}

SS2 biofilm formation required the presence of fibrinogen in the culture medium (Freeman et al., 1989; Bojarska et al., 2016). For SS2 biofilm formation in vitro, $100 \mu \mathrm{l}$ of THB with $2.5 \mathrm{mg} / \mathrm{ml}$ of human plasma fibrinogen (Sigma Aldrich) and $100 \mu \mathrm{l}$ of the bacterial suspension at a concentration of $10^{6}$ colony forming units $(\mathrm{CFU}) / \mathrm{ml}$ were incubated in a 96 -well plate at $37^{\circ} \mathrm{C}$ for $24 \mathrm{~h}$. Each well was washed carefully with PBS to remove planktonic bacteria. For biofilm experiments, the biofilms were resuspended in PBS by repeated pipetting, through which the biofilms were 
physically dispersed (Johnson et al., 2016). To detect the effect of neutrophils on SS2 biofilm formation, $100 \mu \mathrm{l}$ of a $10^{4} \mathrm{CFU} / \mathrm{ml}$ bacteria suspension in RPMI was mixed with $100 \mu$ l of purified neutrophils at $10^{6} / \mathrm{ml}$ with or without DNase I or cytochalasin B in a 96 -well plate and incubated for $24 \mathrm{~h}$ at $37^{\circ} \mathrm{C}$ in $5 \% \mathrm{CO}_{2}$. The purified neutrophils without bacteria were included as a negative control. DNase I and cytochalasin B were added to bacterial suspension only in the presence of fibrinogen to evaluate the influence of these two inhibitors on biofilm formation.

Biofilm formation in the above assay was detected in a 96well plate using a $0.1 \%$ crystal violet stain (Kosikowska et al., 2016). After incubation for $24 \mathrm{~h}$, the plates were washed three times with PBS to remove nonadherent cells. To each well, $200 \mu \mathrm{l}$ of methyl alcohol was added to fix the cells, and then the plates were placed in a $37^{\circ} \mathrm{C}$ dryer oven to remove the methyl alcohol. After the plates were washed with PBS three times, the biofilm in each well was stained with $200 \mu$ l of $0.1 \%$ crystal violet for $20 \mathrm{~min}$. Following staining, the plates were washed three times, and the crystal violet staining the cells was dissolved with $95 \%$ ethyl alcohol. The biofilm was detected with a multifunctional microplate reader (Tecan Infinite Pro, Austria) at an optical density (OD) of $595 \mathrm{~nm}$.

\section{Biofilm Detection In vivo}

To determine whether SS2 forms biofilms in vivo, the bacteria were grown to an $\mathrm{OD}_{600}$ of 0.8 and were then washed three times with PBS. Four-week-old ICR mice were challenged with SS2 ZY05719 at $10^{8} \mathrm{CFU} / \mathrm{ml}$ by intraperitoneal injection. Three days post-injection, the mice were challenged again. At $12 \mathrm{~h}$ after the injection, the mice were euthanized, and the heart, liver, spleen, lungs, and kidneys were collected and homogenized. SS2 biofilm formation was determined using plate streaking in modified Congo Red THB plate agar. In order to exclude the color change of Congo Red THB plate is caused by planktonic bacteria or tissue homogenate, planktonic ZY05719 was added into organs homogenate of non-injected mice and the mixture was streaked on Congo Red THB plate directly. The bacteria isolated in vivo were detected with polymerase chain reaction (PCR) using the primer combinations GAPDHF/GAPDGR to detect SS2 GAPDH: forward, 5' -CATGGACAGATAAAGATGG-3'; reverse, $5^{\prime}$-GCAGCGTATTCTGTCAAACG- $3^{\prime}$ and CPSF/CPSR to detect the SS2 serotype: forward, $5^{\prime}$-GACGGCAACATTGTTGAGTC3'; reverse, 5' -CTCCTAACCACTGTTCAGTG-3'.

\section{Phagocytosis Assay}

The phagocytosis assay was performed with RAW264.7 cells as previously described with some modifications (Mitterstiller et al., 2016). Briefly, RAW264.7 cells were incubated in 24well plates, and then the cell monolayers were washed three times with PBS. An aliquot $(100 \mu \mathrm{l})$ of suspension containing $10^{6} \mathrm{CFU} / \mathrm{ml}$ planktonic cells or biofilm cells were added to the cells. The 24 -well plate was centrifuged at $800 \times \mathrm{g}$ for $10 \mathrm{~min}$ and was incubated for $2 \mathrm{~h}$ at $37^{\circ} \mathrm{C}$ in $5 \% \mathrm{CO}_{2}$. Next, the cells were washed with DMEM and were treated with $200 \mu \mathrm{g} / \mathrm{ml}$ of penicillin-streptomycin for $1 \mathrm{~h}$ to kill extracellular bacteria. The cells were washed with DMEM, and then $100 \mu \mathrm{l}$ of trypsin and $900 \mu \mathrm{l}$ of sterile deionized water were added per well to release the bacteria. The viable bacteria number was determined by plating serial dilutions. Bacteria incubated in DMEM for $2 \mathrm{~h}$ without RAW264.7 cells were served as control group to quantify the initial inoculum. The level of phagocytosis was calculated as (CFUs of viable bacteria in experimental group)/(CFUs of viable bacteria in the control group).

\section{Neutrophil and NET Bactericidal Assays}

The neutrophils bactericidal assay was performed according to a previous method with slight modifications (Uchiyama et al., 2015). The neutrophils were divided into 3 groups: an untreated group containing only purified neutrophils, and two groups of neutrophils were treated with either DNase I (Sigma Aldrich) to inhibit NET formation or with cytochalasin B (Sigma Aldrich) to suppress neutrophil phagocytosis. Bacteria at $3 \times 10^{7} \mathrm{CFU}$ were added to the neutrophils at a multiplicity of infection (MOI) of 10. After incubation with planktonic SS2 or biofilm cells for 90 $\mathrm{min}$, the neutrophils were permeabilized with $0.2 \%$ Triton X-100 (Sigma Aldrich) on ice to release the intracellular bacteria. The surviving bacteria were diluted and plated on THB agar, and the CFUs were counted. Bacteria without incubation were serially diluted and plated to quantify the initial inoculum.

The neutrophils were stimulated by PMA (200 nM, Sigma Aldrich) for $4 \mathrm{~h}$ to form NETs as previously described (Ma et al., 2017). Thereafter, the mixtures were centrifuged at $800 \times \mathrm{g}$ for $10 \mathrm{~min}$ to remove the cells. Planktonic SS2 and biofilm cells at $2 \times 10^{7} \mathrm{CFU}$ were added to the NET supernatant and were incubated for $1 \mathrm{~h}$ at $37^{\circ} \mathrm{C}$ in $5 \% \mathrm{CO}_{2}$. The bacteria without incubation in NETs supernatant were diluted and plated on THB agar as a control.

\section{Bacterial Survival in Mouse Blood In vivo}

Bacterial survival in the blood was determined as previously described (Derkaoui et al., 2016). An aliquot $(200 \mu \mathrm{l})$ of planktonic or biofilm SS2 at an $\mathrm{OD}_{600}$ of 0.5 was injected into mice via the tail vein route. To further evaluate the function of NETs, the bacteria were injected into the tail vein with DNase I (10 mg/kg of body weight), and at $12 \mathrm{~h}$ post-infection, DNase I was injected again. Planktonic and biofilm SS2 cells without incubation were plated on THB agar as a control. At 2, 4, 8, and $24 \mathrm{~h}$ post-infection, blood was collected via heart puncture, and the blood was serially diluted and plated on THB agar plates.

\section{Visualization and Quantification of NETs In vitro}

NETs were observed in vitro as previously described (Yost et al., 2016). Briefly, neutrophils were pretreated with cytochalasin B for 15 min before incubation with PMA and bacteria. Planktonic ZY05719 at an $\mathrm{OD}_{600}$ of $0.6-0.8$ were washed twice with PBS, added to the neutrophils at an MOI of 10 on poly-L-lysine-coated cover slides, and then centrifuged at $800 \times \mathrm{g}$ for $10 \mathrm{~min}$. After incubation at $37^{\circ} \mathrm{C}$ for 3 $\mathrm{h}$, the cover slides were fixed with $4 \%$ paraformaldehyde for $10 \mathrm{~min}$, permeabilized with $0.1 \%$ Triton $\mathrm{X}-100$ and were then blocked with donkey serum at $4^{\circ} \mathrm{C}$ overnight. The samples were stained with the primary rabbit anti-neutrophil histone H4 antibody (citrulline 3, 1:1000 diluted, Merck 
Millipore, Billerica, MA, USA) for $1 \mathrm{~h}$ at RT, followed by incubation with goat anti-rabbit Alexa 568 antibody (1:100 dilution, Jackson ImmunoResearch, West Grove, PA, USA). The DNA was visualized by staining with 4,6-diamidino-2phenylindole (DAPI, Thermo Fisher). The images were recorded using a fluorescence microscope (Zeiss, Germany). Bacteria entrapped by NETs were stained with SYTO 9 green fluorescent nucleic stain (Thermo Fisher) and observed using $100 \times$ oil objective.

The NET quantification assay was performed as previously described (Riyapa et al., 2012). For determining the capacity of neutrophils to form NETs in the presence of planktonic and biofilm SS2, $200 \mu \mathrm{l}$ of neutrophils were incubated with $20 \mu \mathrm{l}$ of planktonic SS2 cells, biofilm cells or bacteria separated from biofilm matrix with or without PMA for $3 \mathrm{~h}$. The biofilms were disrupted and the mixture was centrifuged at 3,000 $\times g$ for $10 \mathrm{~min}$ to separate the bacteria from biofilm matrix. The supernatant was biofilm extracellular matrix and was collected. The precipitate was bacteria that were separated from matrix and the bacteria were washed 3 times with PBS. As a positive control, neutrophils were stimulated with $200 \mathrm{nM}$ PMA. The corresponding bacteria or biofilm matrix were incubated in media without neutrophils to eliminate the background fluorescence. The negative control was the purified neutrophils incubated in media. Extracellular DNA was used to evaluate the quantity of NETs, which was quantified using a Quant-iT Picogreen dsDNA assay kit (Invitrogen). Briefly, after incubation, the reaction mixture were centrifuged at $800 \times \mathrm{g}$ for $5 \mathrm{~min}$ to discard the cells. Subsequently, $100 \mu \mathrm{l}$ of supernatant was added to $100 \mu \mathrm{l}$ of a working solution, which was then mixed thoroughly. After incubation for $5 \mathrm{~min}$, the fluorescence was read with a multifunctional microplate reader (Tecan Infinite Pro) at $480 \mathrm{~nm}$ (excitation)/520 nm (emission).

\section{Statistical Analysis}

All experiments were repeated at least 3 times. Student's $t$-test and the GraphPad Prism 5 Software package (GraphPad Software, La Jolla, CA, USA) were used to perform statistical analyses. Values of $p<0.05$ were considered statistically significant.

\section{RESULTS}

\section{SS2 Biofilm Formation Improved in the Presence of Neutrophils}

Wild-type SS2 can form biofilms only in the presence of fibrinogen in vitro; however, we found that wild-type SS2 can form biofilms without fibrinogen in the presence of neutrophils. Either phagocytosis or NETs of neutrophil is inhibited, the formation of SS2 biofilm decreased (Figures 1A,B). DNase I and cytochalasin B were used to degrade NET DNA and inhibit phagocytosis and these two inhibitors had no influence on SS2 biofilm formation in the presence of fibrinogen (Figure 1C). This results suggest that in the presence of neutrophil infiltration, SS2 is more liable to form biofilms, which may enhance bacterial survival.

\section{Identification of SS2 Biofilms in Mouse Organs}

Considering that SS2 biofilm formation in THB requires fibrinogen, it is necessary to determine whether SS2 forms biofilms in vivo. Biofilm formation can cause a color change of the Congo Red Agar from red to black. Importantly, biofilm SS2 was isolated from the liver, spleen, and kidney of healthy mice challenged with planktonic SS2 (Figure 2A). Control groups are designed to evaluate the influence of planktonic bacteria and tissue homogenate on Congo Red Agar, and the results showed that normal tissue homogenate, planktonic SS2 and even their simple mixtures cannot cause a color change of the Congo Red Agar (Figure 2B). The isolated biofilm cells were confirmed by PCR (Figure 2C). These results indicated that SS2 could form bacterial biofilms during the process of infection.

\section{Chemotaxis of Neutrophils to the Site of Infection Site with Planktonic and Biofilm Cells}

The mice were infected with biofilms and planktonic SS2 at the same $\mathrm{OD}_{600}$ using the murine peritoneal infection model; then, mouse immune cells were collected from peritoneal lavage fluids. Cells collected from mice without infection were served as blank control (Figure 3A). The results indicated that both planktonic and biofilm cells caused a significant increase in neutrophils in the peritoneal cavity, from 2.4 to $87.5 \%$ and $76.2 \%$, respectively (Figures 3B,C). The neutrophil infiltration provides an environment for the interaction between neutrophils and pathogens.

\section{Phagocytosis Efficiency of Biofilm Cells and Planktonic Cells}

The results of the phagocytosis assay indicated that approximately $40 \%$ of planktonic SS2 can be engulfed by RAW246.7 cells, a type of professional phagocyte. However, only a few biofilm cells could be plated on THB agar, which indicated that it was more difficult to engulf biofilm SS2 (Figure 4A). Purified neutrophils had a significant bactericidal effect, and the survival capability of biofilm SS2 was nearly twice that of planktonic SS2. When DNase I was added with neutrophil, the survival rate of planktonic bacteria were nearly 2 times greater than that of the corresponding untreated group. The bacterial survival rate in the biofilm group with DNase I treatment was nearly $30 \%$ higher than that of the corresponding untreated control group. For planktonic SS2, when neutrophil phagocytosis was suppressed, the survival rate of planktonic SS2 was increased significantly. However, regardless of phagocytosis inhibition, there was little influence on the survival capability of the biofilm cells (Figure 4B). Importantly, the results showed that the inhibition of phagocytosis was more beneficial for the survival of planktonic SS2 than the degradation of NETs, while NETs appear to play an important role in biofilm SS2 elimination.

\section{NETs Bactericidal Activity}

To evaluate the bactericidal capacity of NETs, the NETs bactericidal assay was performed. In the presence of NETs, 
A biofilm quantification in neutrophils

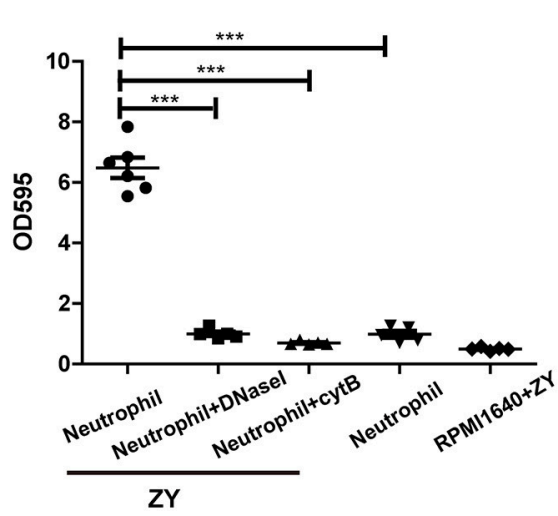

B biofilm formation in neutrophils

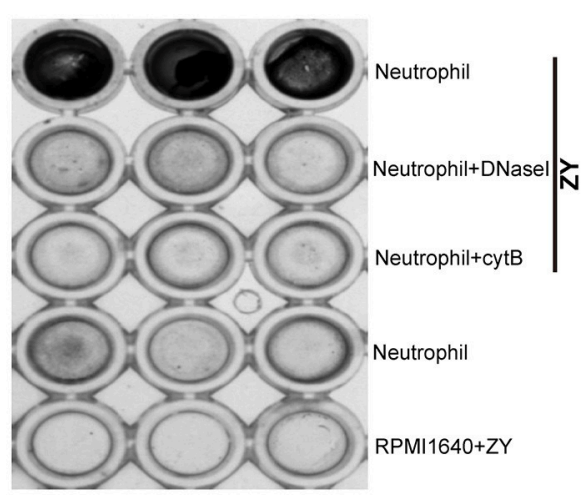

C biofilm formation with frbrinogen

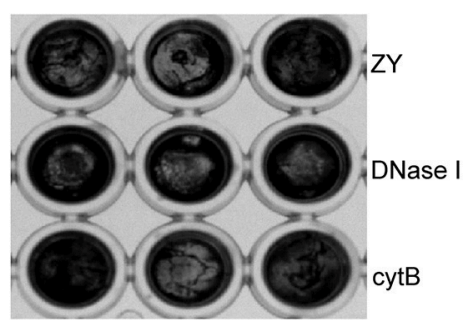

FIGURE 1 | Neutrophil impact on SS2 biofilm formation in vitro. SS2 ZY05719 (referred as ZY in figures) biofilm quantification using crystal violet staining.

(A) The biofilms were quantified using a multifunctional microplate reader at OD 595. When DNase I and cytochalasin B (referred as cytB in figures) were added, the biofilm formation capability decreased up to five-fold. The results are depicted as the mean $\pm \operatorname{SD}(n=5)$. ${ }^{* *} p<0.001$. (B) The corresponding photos of biofilms stained with crystal violet are shown. (C) The influence of DNase I and cytochalasin B on SS2 biofilm formation in the presence of fibrinogen. These two inhibitors have no effect on SS2 biofilm formation.

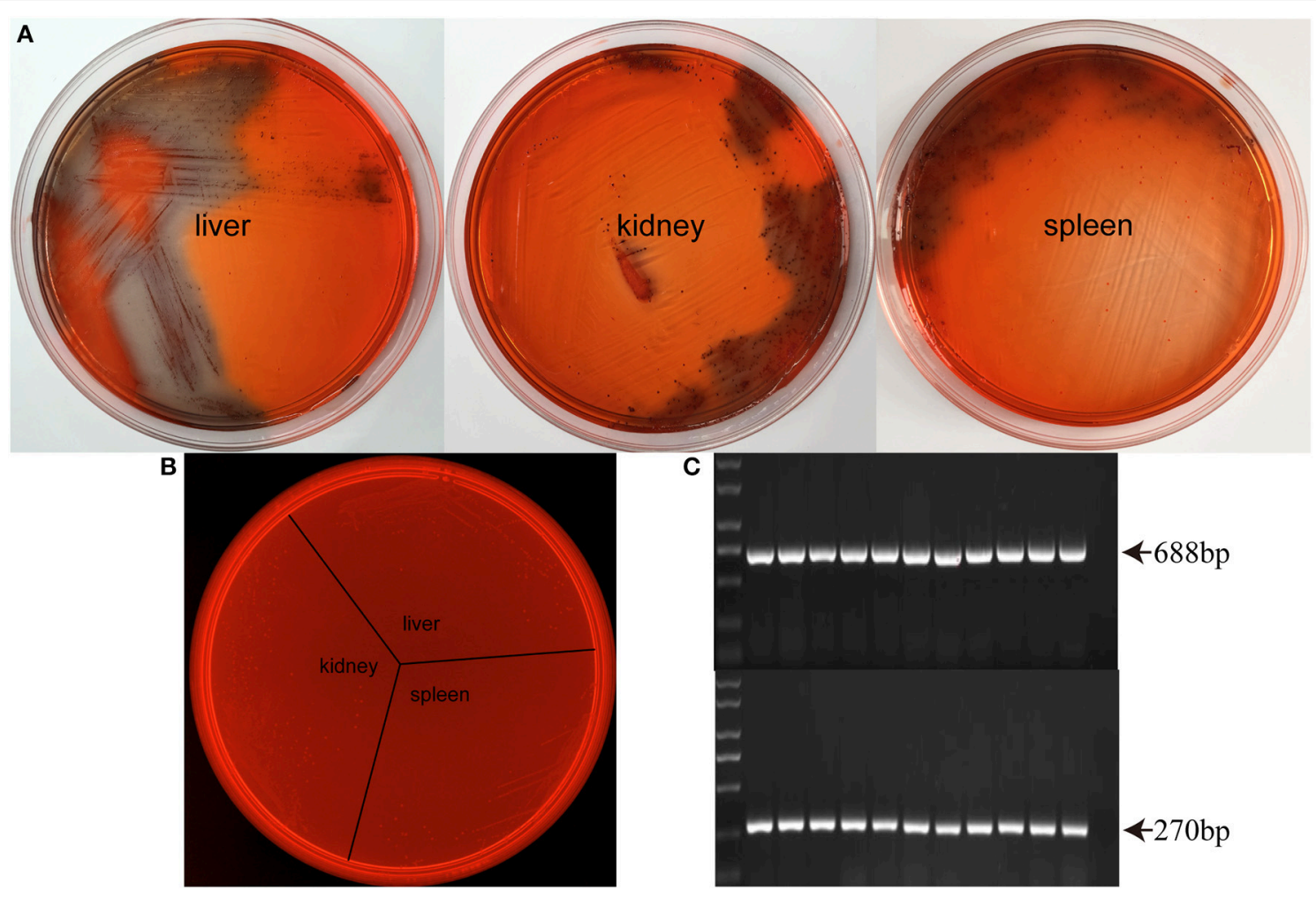

FIGURE 2 | Biofilm formation in SS2 infected mice. (A) At $12 \mathrm{~h}$ after the second injection with SS2 ZY05719, the mouse organs were homogenized, and the bacterial colonies were examined on modified Congo Red THB agar. Biofilm cells isolated from the liver, kidney and spleen can cause a red to black color change. (B) Organs collected from healthy mice were homogenized and mixed with planktonic SS2 ZY05719. The mixtures were streaked on Congo Red THB agar. Planktonic bacteria, tissue homogenate and their simple mixtures cannot cause a color change. (C) Isolated bacteria were confirmed as SS2 by PCR. A PCR product of 688-bp indicated the presence of SS2 GAPDH, and the 270-bp product indicated the presence of the SS2 capsule.

nearly $25 \%$ of planktonic and $20 \%$ of biofilm SS2 cells were killed according to viable bacteria quantification on THB agar (Figure 5). The result indicated that NETs could kill both planktonic and biofilm SS2 and the bactericidal efficiency of NETs on planktonic SS2 and biofilm cells was comparable. 

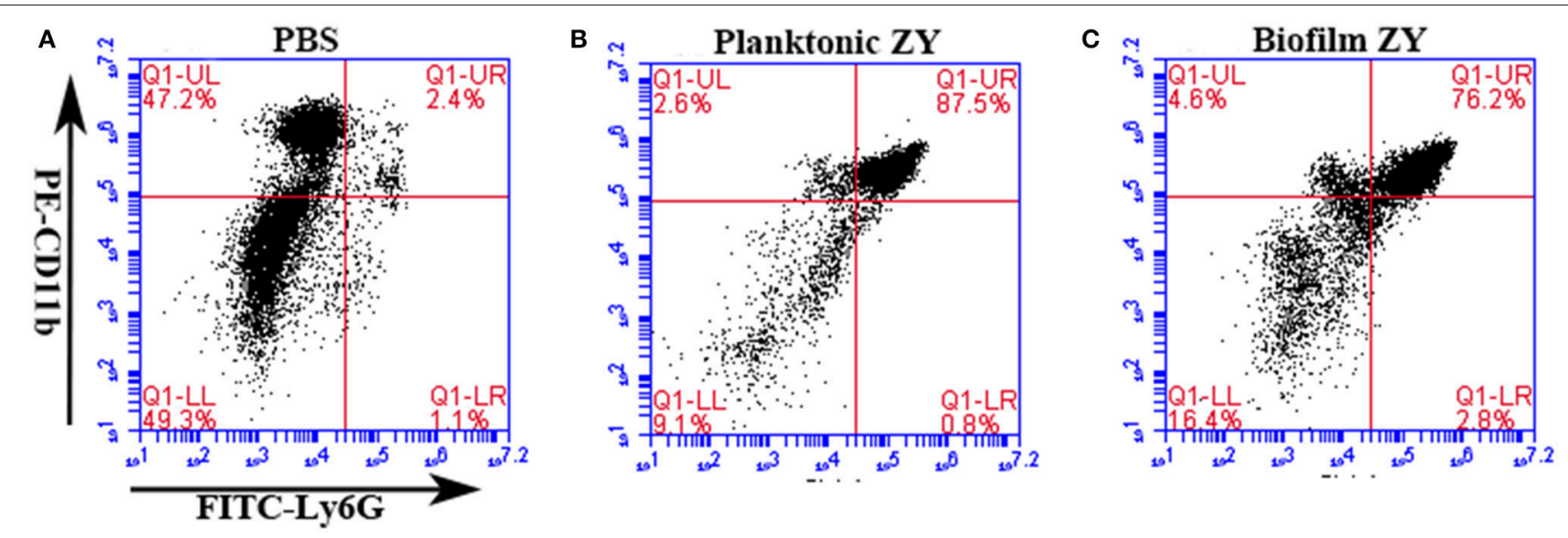

FIGURE 3 | Proportion of neutrophils in peritoneal washes induced by planktonic and biofilm SS2. Neutrophils were marked with PE-conjugated anti-CD11b antibody and FITC-conjugated anti-Ly6G antibody. (A) Neutrophils comprised $2.4 \%$ of the cells collected from mice injected with the PBS control. (B,C) Neutrophils comprised 87.5 and $76.2 \%$ of cells collected from mice infected with planktonic and biofilm SS2, respectively.
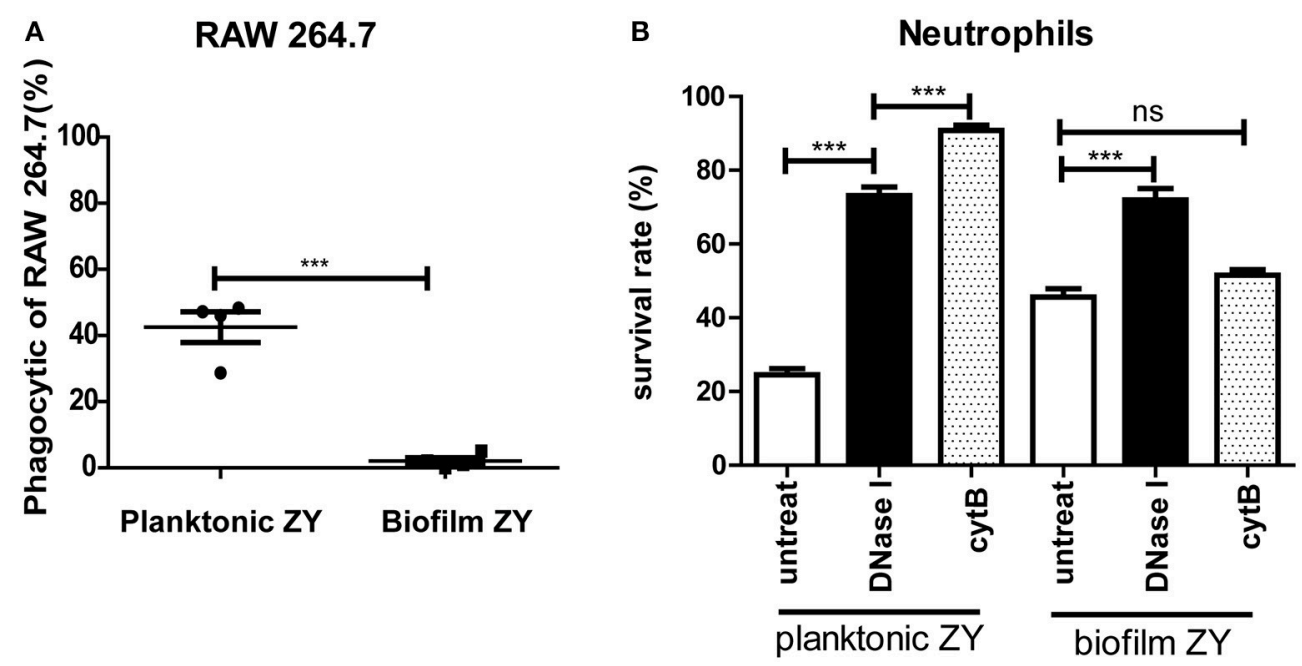

FIGURE 4 | Phagocytosis by RAW264.7 cells and bactericidal activity of neutrophils. (A) Planktonic and biofilm SS2 cells were phagocytized by RAW264.7, and $40 \%$ of planktonic SS2 cells were phagocytized; however, few biofilm SS2 cells were phagocytized. (B) Planktonic and biofilm SS2 cells were killed by neutrophils, and the survival rate of biofilm cell was almost twice that of planktonic cells. When neutrophils were treated with DNase I and cytochalasin B, the survival rate of planktonic cells were increased by 2 -fold and 3-fold, respectively. When neutrophils were treated with DNase I, the viable biofilm cells increased significantly; however, when neutrophils were pretreated with cytochalasin B, there was no significant difference between the untreated control and pretreatment groups. The results are depicted as the mean $\pm \mathrm{SD}(n=5) .{ }^{* \star \star} p<0.001$; ns, no difference between the groups.

\section{Inhibition of NET Formation by SS2 Biofilm}

Because NET formation is an important mechanism to kill SS2 biofilm, we next examined whether SS2 biofilms induced NET formation by neutrophils. SS2 ZY05719 could induce NETs release and could be captured by the NETs (Figures 6A,B).

To further study the influence of biofilms on NET formation, the NETs quantification assay was developed. Only planktonic SS2 and bacteria separated from biofilm extracellular matrix could stimulate NETs release compared to biofilm cells. Importantly, the supernatant of the dispersed biofilm mixture, which is mainly composed of biofilm extracellular matrix, could not stimulate NET formation. In this case, bacteria were incubated with PMA stimulated neutrophils to determine whether biofilms failed to activate neutrophils or inhibit NET formation. The extracellular DNA of NETs induced by planktonic SS2 and bacteria separated from biofilm matrix was enhanced by PMA; however the biofilms and biofilm extracellular matrix inhibited PMA-induced NETs as well (Figure 6C). These results indicated that bacteria both from the planktonic state and the dispersed biofilm state can stimulate NETs release; however, the extracellular biofilm matrix inhibited NET formation. 


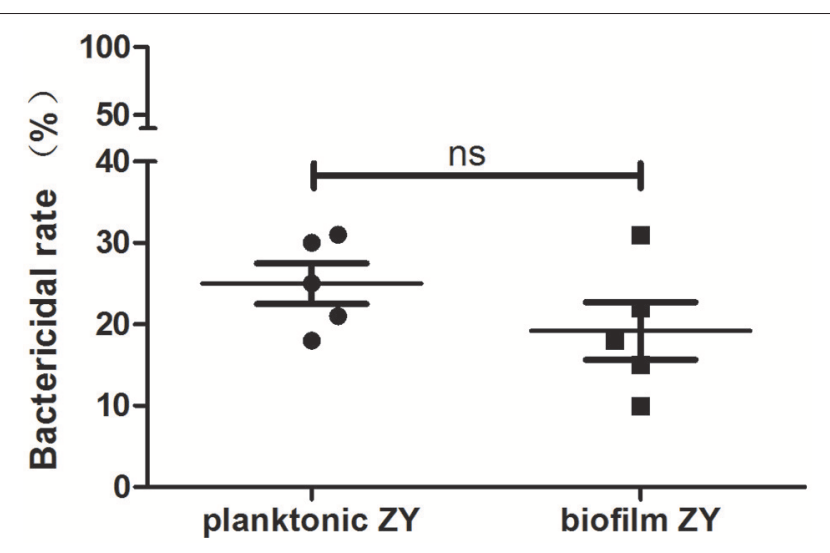

FIGURE 5 | Bactericidal capability of NETs. The bactericidal rate was calculated by viable bacteria quantification on THB agar. The results are depicted as the mean \pm SD $(n=5)$. ns, no difference between groups.

\section{Survival of Planktonic and Biofilm SS2 In vivo}

Following the observation that NETs kill both planktonic SS2 and biofilm SS2 in vitro, we aimed to identify the function of NETs in blood in vivo. In the first $8 \mathrm{~h}$ post-infection, both viable planktonic SS2 and viable biofilm SS2 cells decreased. This result may be ascribe to the host immune response. However, biofilm SS2 survived better than the planktonic cells in the blood stream. Notably, the survival of planktonic and biofilm SS2 was enhanced when NETs were degraded by DNase I. When NETs were destroyed with DNaseI, The viable bacteria in biofilm was much more than planktonic bacteria after $2 \mathrm{~h}$ post-infection (Figure 7 ). These results confirmed that biofilm SS2 demonstrated enhanced survival in the host, particularly when NET DNA was degraded.

\section{DISCUSSION}

SS2 is an important emerging zoonotic pathogen in humans (Smith et al., 2001; Goyette-Desjardins et al., 2014). Most of SS2 human invasive isolates formed biofilm according to previous studies (Bojarska et al., 2016). Bacterial biofilm formed on host surfaces, which is critical to the virulence of these organisms. Neutrophils initiate potent response to evasive pathogens and surveille the tissue in the circulation, which plays an essential role in innate immunity (NicolasAvila et al., 2017). These cells rapidly react to infection and clear pathogens. To date, little information is available on the interaction between neutrophil and SS2. It is critical to study the response of neutrophils to SS2. In this study, we examined the interaction between SS2 and neutrophils and SS2 biofilm formation was increased in the presence of neutrophils. SS2 biofilms can mediate neutrophil phagocytosis evasion; however, SS2 biofilms can be killed by NETs, and the bactericidal efficiency is comparable to the action of NETs on planktonic SS2. Importantly, SS2 biofilm cells can inhibit
NET formation, mainly because of the biofilm extracellular matrix.

Bacterial biofilms can exist in a range of host tissues in the process of bacterial infection, which enables the bacterial communities to persist in the host (Boles and Horswill, 2011). From the perspective of the bacteria and host immune system relationship, pathogens form biofilms to increase chances of survival and to cause persistent infection in the host (Watters et al., 2016). For example, biofilm formation provides pneumococci with a protected environment for bacterial cells and enables transmission from person to person during nasopharyngeal colonization (Marks et al., 2013). Human neutrophils can enhance the development of Pseudomonas aeruginosa biofilms (Walker et al., 2005). In addition, previous studies have reported that macrophages and monocytes increase C. albicans biofilm formation (Chandra et al., 2007; Watters et al., 2016). In this study, the results showed that neutrophils can promote SS2 biofilm formation and that biofilm SS2 was better able to survive than planktonic cells in macrophages and neutrophils in vitro and in blood in vivo. To survive in the host, resistance to phagocytes in the blood is a crucial event for the pathogenicity of SS2 (Zhu et al., 2016). These findings suggest that biofilm formation is a survival strategy utilized by SS2 to evade phagocytosis. In addition, our results demonstrate that SS2 can form biofilms in some tissues such as the liver, spleen and kidney in vivo.

In addition to phagocytosis, neutrophils release NETs to trap and kill pathogens through extracellular DNA and antimicrobial proteins (Thammavongsa et al., 2013). Various pathogens can be killed by NETs including parasites, fungi, bacteria, and viruses (Saitoh et al., 2012; Uchiyama et al., 2015; Avila et al., 2016; Von Kockritz-Blickwede et al., 2016). It has previously been reported that a microbe size-sensing mechanism allows neutrophils to selectively respond to pathogens on the basis of microbe size. Small microbes are more likely to be taken up in a phagolysosome instead of stimulating NET formation (Branzk et al., 2014). One study showed that $C$. albicans with hyphae, which are too large to be phagocytized, are large enough to induce NET formation; however, C. albicans in yeast form failed to induce NETs release and C. albicans biofilms impaired NET formation (Branzk et al., 2014; Johnson et al., 2016). C. albicans biofilms consist of two main kinds of cells, small oval yeast-form cells and long tubular hyphal cells, and both yeast cells and hyphae are crucial for biofilm formation. SS2 and many pathogenic bacteria can induce NET formation. Therefore, virulence mechanisms may have a critical role in NET formation and microbe size may not the most important virulence mechanism that induce NETs in response to bacterial stimuli. SS2 biofilms are communities of bacteria with extracellular DNA, proteins and exopolysaccharides. Importantly, biofilm extracellular matrix can vary greatly depending on the microorganisms present. The different properties between bacterial pathogens and the fungal pathogen C. albicans may contribute to the different activities of biofilms in response to neutrophils. Importantly, phagocytosis is a much faster mechanism than NET formation and phagocytosis remains the major method for host immune cells to clear invasive SS2 cells (Fuchs et al., 2007; Nordenfelt and Tapper, 2011). A 


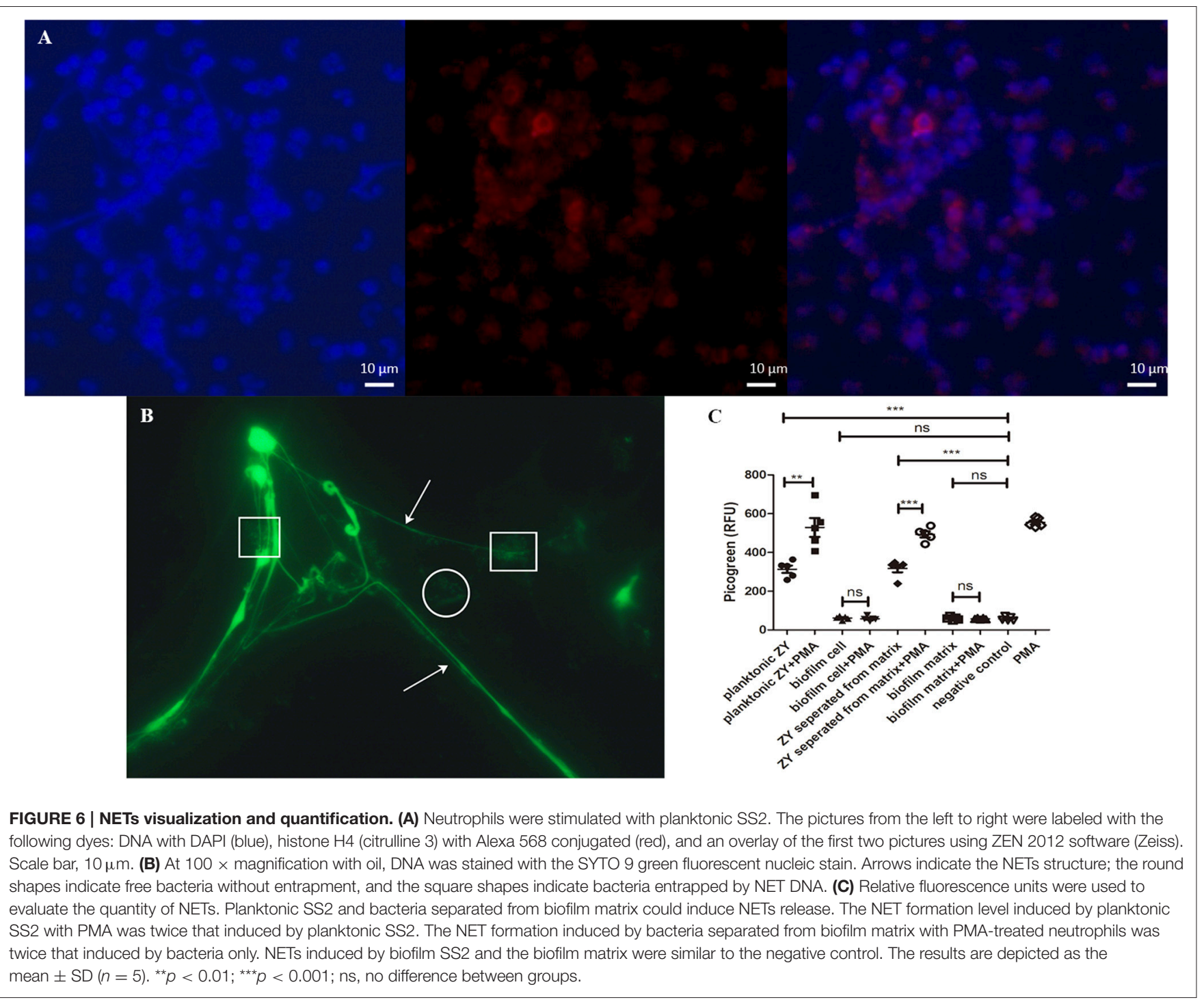

reasonable hypothesis is that NETs aid in the killing of SS2 biofilm cells that are difficult to phagocytose by immune cells and that NETs appear to be an important method of eliminating SS2 biofilm cells.

Both planktonic SS2 and biofilm SS2 cells can cause neutrophil accumulation at infection sites, providing an ideal environment for NETs immunoreaction. Significantly, NETs appear to have equal bactericidal efficacy for biofilm and planktonic SS2 cells. Neutrophils were treated with DNase I and cytochalasin B to degrade NETs and to suppress phagocytosis, respectively. For planktonic SS2, when NETs and phagocytosis were suppressed separately, the bacterial survival in neutrophils was improved significantly. These results indicated that NET formation and phagocytosis are both important mechanisms for killing invasive planktonic SS2, which is consistent with previous reports (De Buhr et al., 2017). Planktonic bacteria were more likely to be cleared by phagocytosis. When NET DNA was degraded, the survival of SS2 biofilm cells increased; however, phagocytosis had no obvious bactericidal effect on biofilm bacteria. In addition, in blood survival assays in vivo, biofilm cells were better able to survive compared to planktonic cells. When NET DNA was degraded, biofilms protected the bacteria from being killed and biofilm cells had enhanced survival in vivo, indicating that NETs could be an important bactericidal mechanism to entrap and kill bacteria biofilms in the host blood stream. Both phagocytosis and NETs are important bactericidal mechanisms for planktonic cells, and planktonic SS2 can stimulate NETs release and can be entrapped by NETs. NET formation appeared to be an efficient bactericidal mechanism for biofilm cells in this study; however, bacterial biofilms and the biofilm extracellular matrix could inhibit NET formation even in the presence of PMA, indicating that biofilms inhibit NETs release mainly through the extracellular matrix. Notably, bacteria separated from biofilms matrix still have the ability to induce NET formation. Further work will address on the mechanism of NET inhibition through biofilm matrix.

Secretion of nuclease has been the main strategy to degrade the NET DNA backbone for bacteria in previous studies (Uchiyama 


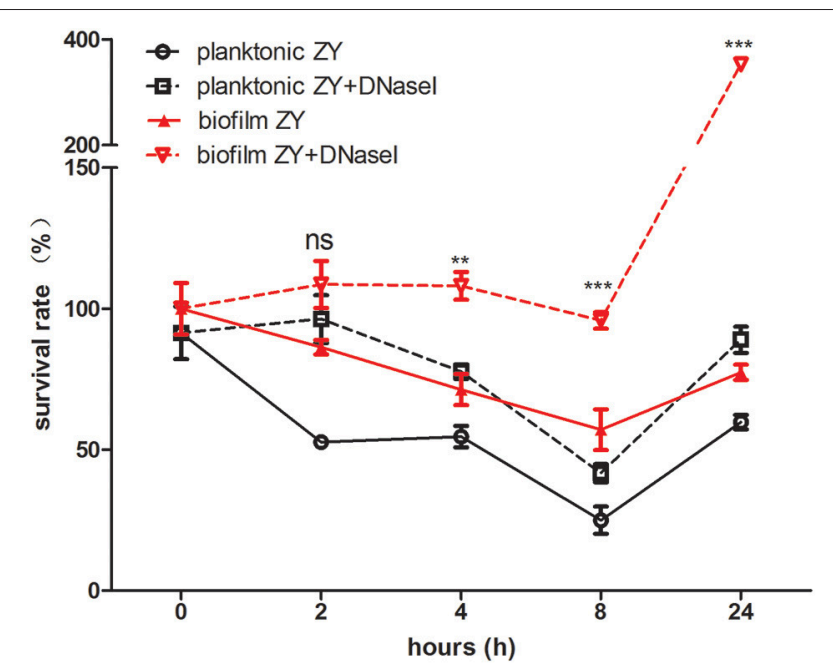

FIGURE 7 | Bacteria survival rate in mouse blood. A comparison of the survival rate of bacteria isolated from mouse blood post-infection between planktonic and biofilm SS2 cells is shown. At 2, 4, 8, and $24 \mathrm{~h}$ post-infection, there were significantly more viable biofilm SS2 cells than planktonic SS2 cells. When DNase I was added to degrade NET DNA, the bacteria both in planktonic state and biofilm state displayed enhanced survival in the blood, particularly for biofilm SS2 cells. The survival rate between the group of planktonic ZY with DNase I and the group of biofilm ZY with DNase I was compared in each time point. The results are depicted as the mean $\pm \mathrm{SD}$ $(n=3) .{ }^{\star \star} p<0.01 ;{ }^{\star * \star} p<0.001$; ns, no difference between groups. and inhibit NET formation, NETs derived from neutrophils stimulated by planktonic bacteria and host inflammatory factors might be a significant mechanism of eliminating bacterial biofilms. This study provides novel knowledge on the battles between NETs and bacterial biofilms and can potentially inform novel strategies for the clinical treatment of streptococcal disease.

\section{AUTHOR CONTRIBUTIONS}

All authors have significant contributions to the completion of the manuscript. Conception and design of the work: FM and HF; acquisition and analysis the data: FM, NY, and GW; interpretation of data for the work: FM, LY, NY, and HF; drafting and revising the work: FM, ZM, HL, and HF; final approval and agreement to be accountable for all aspects of the work: FM, LY, NY, GW, ZM, HL, and HF.

\section{FUNDING}

This study was supported by grants from the National Transgenic Major Program (2014ZX0800946B), the Special Fund for Agroscientific Research in the Public Interest (201403054), the National Natural Science Foundation of China (31272581, 31672574), the Jiangsu Agriculture Science and Technology Innovation Fund $(\mathrm{CX}(16) 1028)$, and the Priority Academic Program Development of Jiangsu Higher Education Institutions (PAPD).

\section{ACKNOWLEDGMENTS}

et al., 2012). In this study we found that biofilm is another mechanism to inhibit NETs release. Importantly, SS2 biofilms inhibit NETs release through the biofilm extracellular matrix. Biofilms are significant protective shelters for bacteria and enable survival by allowing the pathogen to persist and resist the host immune system. Although biofilms can evade phagocytosis

\section{REFERENCES}

Avila, E. E., Salaiza, N., Pulido, J., Rodriguez, M. C., Diaz-Godinez, C., Laclette, J. P., et al. (2016). Entamoeba histolytica trophozoites and lipopeptidophosphoglycan trigger human neutrophil extracellular traps. PLoS ONE 11:e0158979. doi: 10.1371/journal.pone.0158979

Barletta, K. E., Cagnina, R. E., Wallace, K. L., Ramos, S. I., Mehrad, B., and Linden, J. (2012). Leukocyte compartments in the mouse lung: distinguishing between marginated, interstitial, and alveolar cells in response to injury. J. Immunol. Methods 375, 100-110. doi: 10.1016/j.jim.2011.09.013

Beiter, K., Wartha, F., Albiger, B., Normark, S., Zychlinsky, A., and HenriquesNormark, B. (2006). An endonuclease allows Streptococcus pneumoniae to escape from neutrophil extracellular traps. Curr. Biol. 16, 401-407. doi: 10.1016/j.cub.2006.01.056

Bojarska, A., Molska, E., Janas, K., Skoczynska, A., Stefaniuk, E., Hryniewicz, W., et al. (2016). Streptococcus suis in invasive human infections in Poland: clonality and determinants of virulence and antimicrobial resistance. Eur. J. Clin. Microbiol. Infect. Dis. 35, 917-925. doi: 10.1007/s10096-016-2616-x

Boles, B. R., and Horswill, A. R. (2011). Staphylococcal biofilm disassembly. Trends. Microbiol. 19, 449-455. doi: 10.1016/j.tim.2011.06.004

Branzk, N., Lubojemska, A., Hardison, S. E., Wang, Q., Gutierrez, M. G., Brown, G. D., et al. (2014). Neutrophils sense microbe size and selectively release neutrophil extracellular traps in response to large pathogens. Nat. Immunol. 15, 1017-1025. doi: 10.1038/ni.2987
We thank flow cytometer unit (Laboratory Center of Life Science, Nanjing Agriculture University), fluorescence microscope and multifunctional microplate reader unit (National Center of Meat Quality and Safety Control, Nanjing Agriculture University).

Brinkmann, V., Reichard, U., Goosmann, C., Fauler, B., Uhlemann, Y., Weiss, D. S., et al. (2004). Neutrophil extracellular traps kill bacteria. Science 303, 1532-1535. doi: 10.1126/science.1092385

Buchanan, J. T., Simpson, A. J., Aziz, R. K., Liu, G. Y., Kristian, S. A., Kotb, M., et al. (2006). DNase expression allows the pathogen group A Streptococcus to escape killing in neutrophil extracellular traps. Curr. Biol. 16, 396-400. doi: 10.1016/j.cub.2005.12.039

Chandra, J., Mccormick, T. S., Imamura, Y., Mukherjee, P. K., and Ghannoum, M. A. (2007). Interaction of Candida albicans with adherent human peripheral blood mononuclear cells increases C. albicans biofilm formation and results in differential expression of pro- and anti-inflammatory cytokines. Infect. Immun. 75, 2612-2620. doi: 10.1128/IAI.01841-06

Csomos, K., Kristof, E., Jakob, B., Csomos, I., Kovacs, G., Rotem, O., et al. (2016). Protein cross-linking by chlorinated polyamines and transglutamylation stabilizes neutrophil extracellular traps. Cell. Death Dis. 7, e2332. doi: 10.1038/ cddis. 2016.200

De Buhr, N., Reuner, F., Neumann, A., Stump-Guthier, C., Tenenbaum, T., Streptococcus suis-infected cerebrospinal fluid compartment. Cell. Microbiol. 19:e12649. doi: 10.1111/cmi.12649

Derkaoui, M., Antunes, A., Poncet, S., Nait Abdallah, J., Joyet, P., Maze, A., et al. (2016). The phosphocarrier protein HPr of Neisseria meningitidis interacts with the transcription regulator CrgA and its deletion affects capsule production, cell adhesion, and virulence. Mol. Microbiol. 100, 788-807. doi: 10.1111/mmi.13349 Schroten, H., et al. (2017). Neutrophil extracellular trap formation in the 
Freeman, D., Falkiner, F., and Keane, C. (1989). New method for detecting slime production by coagulase negative staphylococci. J. Clin. Pathol. 42, 872-874. doi: $10.1136 /$ jcp. 42.8 .872

Fuchs, T. A., Abed, U., Goosmann, C., Hurwitz, R., Schulze, I., Wahn, V., et al. (2007). Novel cell death program leads to neutrophil extracellular traps. J. Cell Biol. 176, 231-241. doi: 10.1083/jcb.200606027

Gomez, E., Kennedy, C. C., Gottschalk, M., Cunningham, S. A., Patel, R., and Virk, A. (2014). Streptococcus suis-related prosthetic joint infection and streptococcal toxic shock-like syndrome in a pig farmer in the United States. J. Clin. Microbiol. 52, 2254-2258. doi: 10.1128/JCM.02934-13

Gottschalk, M., Xu, J., Calzas, C., and Segura, M. (2010a). Streptococcus suis: a new emerging or an old neglected zoonotic pathogen? Future Microbiol. 5, 371-391. doi: $10.2217 / \mathrm{fmb} \cdot 10.2$

Gottschalk, M., Xu, J., Lecours, M.-P., Grenier, D., Fittipaldi, N., and Segura, M. (2010b). Streptococcus suis infections in humans: what is the prognosis for Western countries?(Part II). Clin. Microbiol. Newsl. 32, 97-102. doi: 10.1016/j.clinmicnews.2010.06.001

Goyette-Desjardins, G., Auger, J. P., Xu, J., Segura, M., and Gottschalk, M. (2014). Streptococcus suis, an important pig pathogen and emerging zoonotic agent-an update on the worldwide distribution based on serotyping and sequence typing. Emerg. Microbes. Infect. 3:e45. doi: 10.1038/emi.2014.45

Hakkim, A., Furnrohr, B. G., Amann, K., Laube, B., Abed, U. A., Brinkmann, V., et al. (2010). Impairment of neutrophil extracellular trap degradation is associated with lupus nephritis. Proc. Natl. Acad. Sci. U.S.A. 107, 9813-9818. doi: 10.1073/pnas.0909927107

Johnson, C. J., Cabezas-Olcoz, J., Kernien, J. F., Wang, S. X., Beebe, D. J., Huttenlocher, A., et al. (2016). The extracellular matrix of candida albicans biofilms impairs formation of neutrophil extracellular traps. PLoS Pathog. 12:e1005884. doi: 10.1371/journal.ppat.1005884

Kerdsin, A., Gottschalk, M., Hatrongjit, R., Hamada, S., Akeda, Y., and Oishi, K. (2016). Fatal septic meningitis in child caused by Streptococcus suis Serotype 24. Emerg. Infect. Dis. 22, 1519-1520. doi: 10.3201/eid2208.160452

Kosikowska, U., Rybojad, P., Stepien-Pysniak, D., Zbikowska, A., and Malm, A. (2016). Changes in the prevalence and biofilm formation of Haemophilus influenzae and Haemophilus parainfluenzae from the respiratory microbiota of patients with sarcoidosis. BMC Infect. Dis. 16, 449. doi: 10.1186/s12879-016-1793-7

Leshner, M., Wang, S., Lewis, C., Zheng, H., Chen, X. A., Santy, L., et al. (2012). PAD4 mediated histone hypercitrullination induces heterochromatin decondensation and chromatin unfolding to form neutrophil extracellular trap-like structures. Front. Immunol. 3:307. doi: 10.3389/fimmu.2012.00307

Ma, F., Guo, X., and Fan, H. (2017). Extracellular nucleases of Streptococcus equi ssp. zooepidemicus degrade host neutrophil extracellular traps and impair macrophage activity. Appl. Environ. Microb. 83, e02468-e02416. doi: 10.1128/AEM.02468-16

Marks, L. R., Davidson, B. A., Knight, P. R., and Hakansson, A. P. (2013). Interkingdom signaling induces Streptococcus pneumoniae biofilm dispersion and transition from asymptomatic colonization to disease. MBio 4:e00438-13. doi: $10.1128 / \mathrm{mBio} .00438-13$

May, R. C., Lee, M. J., Liu, H., Barker, B. M., Snarr, B. D., Gravelat, F. N., et al. (2015). The fungal exopolysaccharide galactosaminogalactan mediates virulence by enhancing resistance to neutrophil extracellular traps. PLoS Pathog. 11:e1005187. doi: 10.1371/journal.ppat.1005187

Mitterstiller, A. M., Haschka, D., Dichtl, S., Nairz, M., Demetz, E., Talasz, H., et al. (2016). Heme oxygenase 1 controls early innate immune response of macrophages to Salmonella typhimurium infection. Cell. Microbiol. 18, 1374-1389. doi: 10.1111/cmi.12578

Nicolas-Avila, J. A., Adrover, J. M., and Hidalgo, A. (2017). Neutrophils in homeostasis, immunity, and cancer. Immunity 46, 15-28. doi: 10.1016/ j.immuni.2016.12.012

Nordenfelt, P., and Tapper, H. (2011). Phagosome dynamics during phagocytosis by neutrophils. J. Leukoc. Biol. 90, 271-284. doi: 10.1189/jlb.08 10457

Riyapa, D., Buddhisa, S., Korbsrisate, S., Cuccui, J., Wren, B. W., Stevens, M. P., et al. (2012). Neutrophil extracellular traps exhibit antibacterial activity against burkholderia pseudomallei and are influenced by bacterial and host factors. Infect. Immun. 80, 3921-3929. doi: 10.1128/IAI.00806-12

Saitoh, T., Komano, J., Saitoh, Y., Misawa, T., Takahama, M., Kozaki, T., et al. (2012). Neutrophil extracellular traps mediate a host defense response to human immunodeficiency virus-1. Cell Host Microbe 12, 109-116. doi: 10.1016/j.chom.2012.05.015

Smith, H. E., Buijs, H., Wisselink, H. J., Stockhofe-Zurwieden, N., and Smits, M. A. (2001). Selection of virulence-associated determinants of Streptococcus suis serotype 2 by in vivo complementation. Infect. Immun. 69, 1961-1966. doi: 10.1128/IAI.69.3.1961-1966.2001

Tang, J., Feng, Y., Yang, W., Song, H., Chen, Z., Yu, H., et al. (2006). Streptococcal toxic shock syndrome caused by Streptococcus suis serotype 2. PLoS Med. 3:e151. doi: 10.1371/journal.pmed.0030151

Thammavongsa, V., Missiakas, D. M., and Schneewind, O. (2013). Staphylococcus aureus degrades neutrophil extracellular traps to promote immune cell death. Science 342, 863-866. doi: 10.1126/science. 1242255

Thurlow, L. R., Hanke, M. L., Fritz, T., Angle, A., Aldrich, A., Williams, S. H., et al. (2011). Staphylococcus aureus biofilms prevent macrophage phagocytosis and attenuate inflammation in vivo. J. Immunol. 186, 6585-6596. doi: 10.4049/jimmunol.1002794

Uchiyama, S., Andreoni, F., Schuepbach, R. A., Nizet, V., and Zinkernagel, A. S. (2012). DNase Sdal allows invasive M1T1 Group A Streptococcus to prevent TLR9-dependent recognition. PLoS Pathog. 8:e1002736. doi: 10.1371/journal.ppat.1002736

Uchiyama, S., Dohrmann, S., Timmer, A. M., Dixit, N., Ghochani, M., Bhandari, T., et al. (2015). Streptolysin O rapidly impairs neutrophil oxidative burst and antibacterial responses to Group A Streptococcus. Front. Immunol. 6:581. doi: 10.3389/fimmu.2015.00581

Uhlmann, J., Siemens, N., Kai-Larsen, Y., Fiedler, T., Bergman, P., Johansson, L., et al. (2016). Phosphoglycerate kinase-A novel streptococcal factor involved in neutrophil activation and degranulation. J. Infect. Dis. 214, 1876-1883. doi: 10.1093/infdis/jiw450

Von Kockritz-Blickwede, M., Blodkamp, S., and Nizet, V. (2016). Interaction of bacterial exotoxins with neutrophil extracellular traps: impact for the infected host. Front. Microbiol. 7:402. doi: 10.3389/fmicb.2016.00402

Walker, T. S., Tomlin, K. L., Worthen, G. S., Poch, K. R., Lieber, J. G., Saavedra, M. T., et al. (2005). Enhanced Pseudomonas aeruginosa biofilm development mediated by human neutrophils. Infect. Immun. 73, 3693-3701. doi: 10.1128/IAI.73.6.3693-3701.2005

Wartha, F., Beiter, K., Normark, S., and Henriques-Normark, B. (2007). Neutrophil extracellular traps: casting the NET over pathogenesis. Curr. Opin. Microbiol. 10, 52-56. doi: 10.1016/j.mib.2006.12.005

Watters, C., Fleming, D., Bishop, D., and Rumbaugh, K. P. (2016). Host responses to biofilm. Prog. Mol. Biol. Transl. Sci. 142, 193-239. doi: 10.1016/bs.pmbts. 2016.05.007

Wertheim, H. F., Nghia, H. D., Taylor, W., and Schultsz, C. (2009). Streptococcus suis: an emerging human pathogen. Clin. Infect. Dis. 48, 617-625. doi: $10.1086 / 596763$

Yang, Y. B., Chen, J. Q., Zhao, Y. L., Bai, J. W., Ding, W. Y., Zhou, Y. H., et al. (2016). Sub-MICs of azithromycin decrease biofilm formation of Streptococcus suis and increase capsular polysaccharide content of S. suis. Front. Microbiol. 7:1659. doi: $10.3389 /$ fmicb.2016.01659

Yost, C. C., Schwertz, H., Cody, M. J., Wallace, J. A., Campbell, R. A., Vieira-DeAbreu, A., et al. (2016). Neonatal NET-inhibitory factor and related peptides inhibit neutrophil extracellular trap formation. J. Clin. Invest. 126, 3783-3798. doi: 10.1172/JCI83873

Zhao, J., Pan, S., Lin, L., Fu, L., Yang, C., Xu, Z., et al. (2015). Streptococcus suis serotype 2 strains can induce the formation of neutrophil extracellular traps and evade trapping. Fems Microbiol. Lett. 362:fnv022. doi: 10.1093/femsle/fnv022

Zhu, J., Zhang, T., Su, Z., Li, L., Wang, D., Xiao, R., et al. (2016). (p)ppGpp synthetases regulate the pathogenesis of zoonotic Streptococcus suis. Microbiol. Res. 191, 1-11. doi: 10.1016/j.micres.2016.05.007

Conflict of Interest Statement: The authors declare that the research was conducted in the absence of any commercial or financial relationships that could be construed as a potential conflict of interest.

Copyright (C) 2017 Ma, Yi, Yu, Wang, Ma, Lin and Fan. This is an open-access article distributed under the terms of the Creative Commons Attribution License (CC BY). The use, distribution or reproduction in other forums is permitted, provided the original author(s) or licensor are credited and that the original publication in this journal is cited, in accordance with accepted academic practice. No use, distribution or reproduction is permitted which does not comply with these terms. 